\title{
Electron Density Measurements from Right-Hand Cutoff of ECE in the TCABR Tokamak
}

\author{
A. M. M. Fonseca, R. P. da Silva, R. M. O. Galvão, Yu. K. Kuznetzov, \\ J. I. Elizondo, E. R. Calderon, L. F. Ruchko, and J. H. Vuolo \\ Laboratório de Física de Plasmas \\ Instituto de Física, Universidade de São Paulo, 05315-970, São Paulo, Brazil
}

Received on 4 February, 2004; revised version received on 15 May, 2004

\begin{abstract}
In tokamak machines with low toroidal magnetic fields and high plasma densities, the accessibility conditions impose restrictions to the detection of the Electron Cyclotron Emission (ECE). In these machines, the righthand cutoff condition can be used as an independent method to determine the local electron density from the ECE data in thermal discharges. In this paper is shown the results obatined from the detection of ECE radiation in the TCABR tokamak, in operation at the Institute of Physics of University of São Paulo. The effect of the ECE radiation cutoff was observed for different radial positions of the plasma column. To reach the ECE cutoff condition, the electron density was increased monotonically by the use of an external gas puffing system. For sufficient high densities, the emission at some frequencies is cutoff and the first and the last frequency to be cutoff depends on the shape of the density profile. These measurements do not require the plasma to be optically thick. It was observed that, for a toroidal field $B_{O}=1.14 T$, the first cutoff of ECE occurs for a radial position $r \cong 5 \mathrm{~cm}$. From these measurements the radial electron density was determined. For a symmetric parabolic profile $n_{e}=n_{e o}\left[1-(r / a)^{2}\right]^{\alpha}$, values of $\alpha$ between 0.86 and 0.97 were experimentally obtained. A good agreement of these values with those obtained from the microwave interferometer measurements $(\alpha \approx 0.85)$ was found. Therefore, the ECE right-hand cutoff constitutes an independent method to obtain information about the electron density profile.
\end{abstract}

\section{Introduction}

ECE radiometry is a well established diagnostic for the electron temperature measurements in Tokamak machines [1]. In some cases, when the ECE radiation in X mode attains the cutoff region, temperature measurements cannot be performed, but, in despite of this, the radiometer can be used to obtain information about the density profile. In this paper, we experimentally obtain the electron density from the ECE right-hand cutoff in the TCABR tokamak. This technique was first used by Lohr [2] to determine the electron density in the DIII-D tokamak. Above the minimum value of the electron density, when the ECE signal begins to cutoff, only the frequency used in the ECE radiometer and the magnetic field (neglecting the poloidal magnetic field) are needed to determine the local electron density. This procedure constitutes an independent method to obatin the local electron density and the results can be compared with those obtained with the microwave interferometer. From the value of ECE frequency that is first/last in the cutoff, the electron density profile can be determined, providing that this can be described by only one or two parameters [3].

In Section 2, a simple description of the experimental arrangement used for the measurements in the TCABR is shown. Some theoretical points of ECE cutoff method are discussed in Section 3. In Section 4, the experimental results are presented and discussed.

\section{Experimental aspects related to the ECE measurements}

Measurements of the electron temperature in the TCABR tokamak ( main parameters: Major radius $R_{0}=61.5 \mathrm{~cm}$, Minor radius $a=18 \mathrm{~cm}$, Plasma current $I_{p}<120 \mathrm{kA}$, Plasma current duration $t_{p}<150 \mathrm{~ms}$ and Toroidal magnetic field $B_{0}=1.1-1.2 T$ ) are routinely obtained with a sweeping heterodyne radiometer $(50-85 \mathrm{GHz})$ [4][5]. This system operates in the $\mathrm{X}$ mode second harmonic. The antenna axis is installed on the equatorial plane of the machine and perpendicular to the plasma column axis. The radiometer Intermediate Frequency ( IF ) bandwidth $(\approx$ $2 \mathrm{GHz}$ ) and the Gaussian Antenna determine the spatial resolution of the radiometer. The waist diameter of the radiation beam $(\approx 2 \mathrm{~cm})$ and the focus position (focal lenght $\approx 37 \mathrm{~cm}$ ) of the antenna were experimentally obtained [5].

The electron density profile obtained from the ECE radiation were compared with the results obtained from a microwave interferometer installed in TCABR [6]. The electron density profiles can be approximated by parabolic equation in the form:

$$
n_{e}(r)=n_{e 0}\left[1-\left(\frac{r}{a}\right)^{2}\right]^{\alpha} .
$$




\section{DIAGNOSTIC LAYOUT}

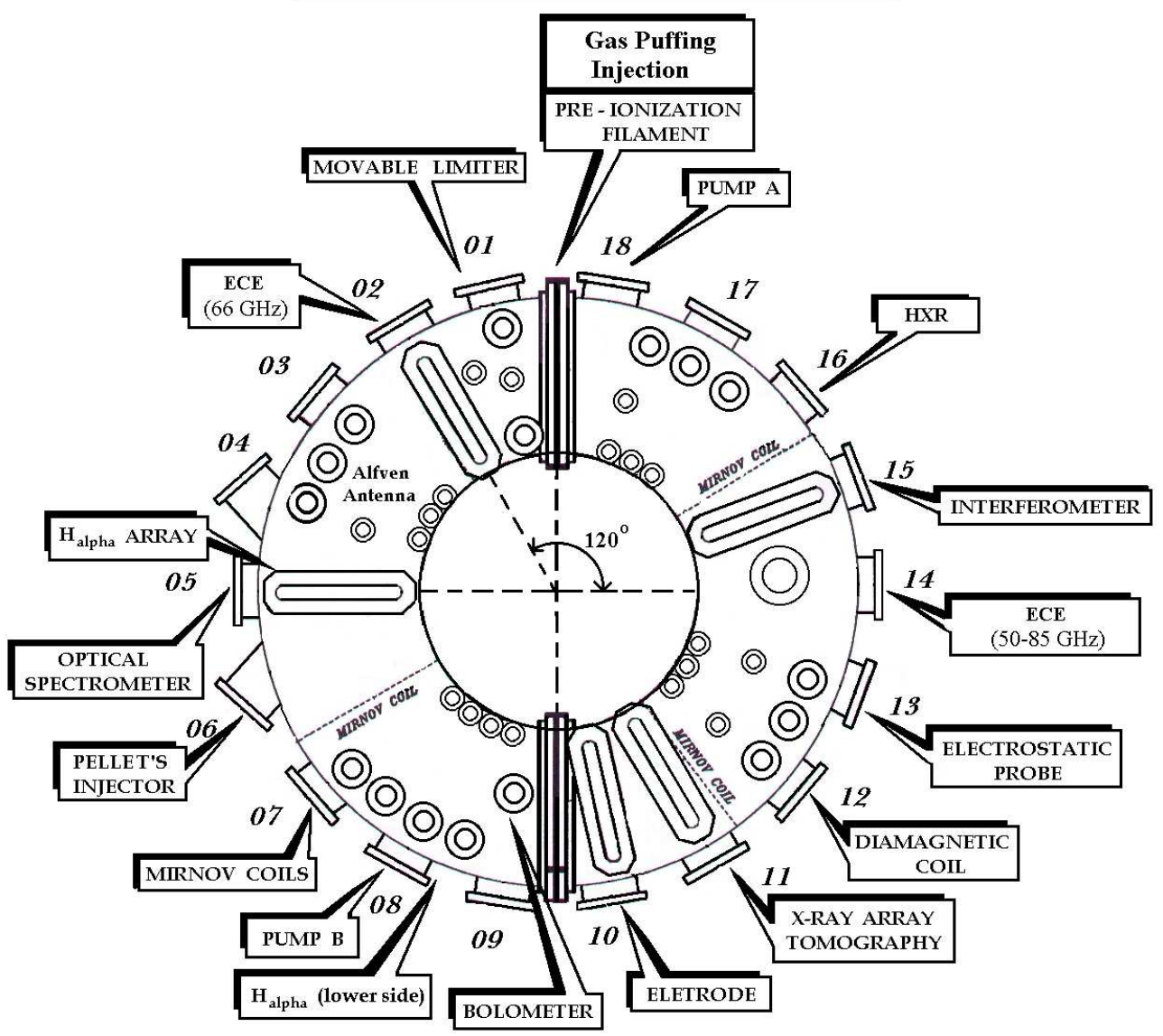

Figure 1. Schematic diagram (top view) showing the location of the main diagnostic of the TCABR tokamak.

A gas puffing injection system is normally used in the TCABR tokamak to control the electron density during the discharges. With this gas puffing system, is possible to built triangular shapes in the time density profiles. As the righthand cutoff frequency becomes equal or larger than the second ECE harmonic, due to the electron density increase, a decrease in the ECE signal is clearly observed.

In Fig. 1, a schematic top view diagram of the TCABR diagnostics location is shown. The ECE sweeping radiometer and the interferometer are installed respectively in a horizontal (window 14 ) and in a vertical (window 15 ) diagnostic port. The gas puffing injection valve is installed in an access port between the windows 1 and 18 .

\section{Method for the $n_{e}$ profile determi- nation from ECE cutoffs}

The accessibility conditions for the ECE radiation in the TCABR tokamak is based on the theory of cold magnetized plasma wave propagation. The cold plasma approximation is valid when the thermal kinetic velocity of the electrons is much less than the phase velocity of the waves. Also the collision effects can be neglected because the collision frequency is much lower than the wave frequency. As the ECE antenna is installed perpendicular to the TCABR minor axis, we consider that the wave vector is perpendicular to the magnetic field. In this case the dieletric tensor is given by $[7]$ :

$$
\overleftrightarrow{\varepsilon}=\left[\begin{array}{ccc}
1-\frac{\omega_{p e}^{2}}{\omega^{2}-\omega_{c e}^{2}} & -i \frac{\omega_{p e}^{2} \omega_{c e}}{\omega\left(\omega^{2}-\omega_{c e}^{2}\right)} & 0 \\
-i \frac{\omega_{p e}^{2} \omega_{c e}}{\omega\left(\omega^{2}-\omega_{c e}^{2}\right)} & 1-\frac{\omega_{p e}^{2}}{\omega^{2}-\omega_{c e}^{2}} & 0 \\
0 & 0 & 1-\frac{\omega_{p e}^{2}}{\omega^{2}}
\end{array}\right]
$$

where the electron cyclotron frequency $\left(\omega_{c e}\right)$ and the plasma frequency $\left(\omega_{p e}\right)$ are given respectively by :

$$
\omega_{c e}=\frac{e B_{t}}{m_{e}}
$$

and

$$
\omega_{c e}=\left(\frac{n_{e} e^{2}}{m_{e} \varepsilon_{o}}\right)^{\frac{1}{2}},
$$

where $e, m_{e}$, are the charge and the mass of the electron, $\varepsilon_{o}$ is the permitivity of free space and $B_{t}$ is the toroidal magnetic field. 
The dispersion relation is obtained calculating the determinant of the matrix :

$$
\overleftrightarrow{\Lambda}=\vec{k} \vec{k}-k^{2} \overleftrightarrow{1}+\frac{w^{2}}{c^{2}} \overleftrightarrow{\varepsilon}
$$

where $\vec{k}$ is the wave vector and $c$ is the light velocity. For a non-trivial solution, the $\operatorname{det} \overleftrightarrow{\Lambda}$ must be equal to zero. The solution for the case where $E_{\perp} \neq 0$, i.e. for $\mathrm{X}$ mode, the dispersion relation is given by

$$
\left(\tilde{n}^{2}\right)_{X}=\frac{\left(\omega^{2}+\omega \omega_{c e}-\omega_{p e}^{2}\right)\left(\omega^{2}-\omega \omega_{c e}-\omega_{p e}^{2}\right)}{\omega^{2}\left(\omega^{2}-\omega_{c e}^{2}-\omega_{p e}^{2}\right)} .
$$

The rigth-hand cutoff frequency is obtained from $\left(\tilde{n}^{2}\right)_{X}=$ 0 and is given by

$$
\omega_{c, R H}=\frac{\omega_{c e}+\left(\omega_{c e}^{2}+4 \omega_{p e}^{2}\right)^{\frac{1}{2}}}{2} .
$$

From this equation we observe that the frequency depends only of the magnetic field $\left(\omega_{c e}\right)$ and the eletron density $\left(\omega_{p e}\right)$. From the right-hand cutoff frequency, the eletron density can be determined from the ECE measurements if we know the magnetic field. To occur a local cutoff we need to have the condition:

$$
\omega_{c R H} \geq 2 \omega_{c e} .
$$

In Fig. 2.a are represented grafically $\omega_{c e}$ and $2 \omega_{c e}$, first and the second ECE harmonics, and $\omega_{c R H}$, right-hand cutoff, for different values of electron density. The density profile, used in this figure, is given by Equation 1 and the toroidal magnetic field was $B_{0}=1.14 T$. The first cutoff occurs when the right-hand cutoff curve touches the second ECE harmonic curve. This happens at the point $a$ for an electron density of $n_{e 0}=2.3 \times 10^{19} \mathrm{~m}^{-3}$ ( at position $r=5.2 \mathrm{~cm}$ ).

In Fig 2.b, the cutoff region is presented in a radius versus density curve. The hachured part of this figure corresponds to the region where ECE cutoff occurs in the TCABR tokamak. As the electron density increases, a larger part of the second harmonic band becomes inacessible. When the cutoff frequency equals the ECE frequency at the same location, the emission in locally cutoff ( see for example the points $a, b, c$ and $e$ in Fig. 2). On other hand, if $\omega_{c R H}$ is larger than the emission frequency, the cutoff frequency occurs non-locally. Note that the emission from the point $d$ to $f$ is not accessible for the density equal to $4.6 \times 10^{19} \mathrm{~m}^{-3}$, when the antenna is located in the low field side.

Substituting the Equation 7 into Equation 8, and considering Equations 3, 4 and 1, we obtain a relation between the the peak density $n_{e 0}, B_{0}, \alpha$ and $r_{f c 0}$, given by :

$$
n_{e 0}=\frac{1.94 \times 10^{19} B_{0}}{\left(1+\frac{r_{f c 0}}{R_{0}}\right)^{2}\left(1-\frac{r_{f c 0}}{a}\right)^{\alpha}} .
$$

By this equation we see that the ECE radiation begins to be inaccessible for a radial position where $d n_{e 0} / d r_{f c 0}=$
0 . Taking the $r$ derivative of Equation 9 and equalizing to zero we obtain :

$$
\alpha=\frac{a^{2}-r_{f c 0}^{2}}{r_{f c 0}^{2}+r_{f c 0} R_{0}}
$$
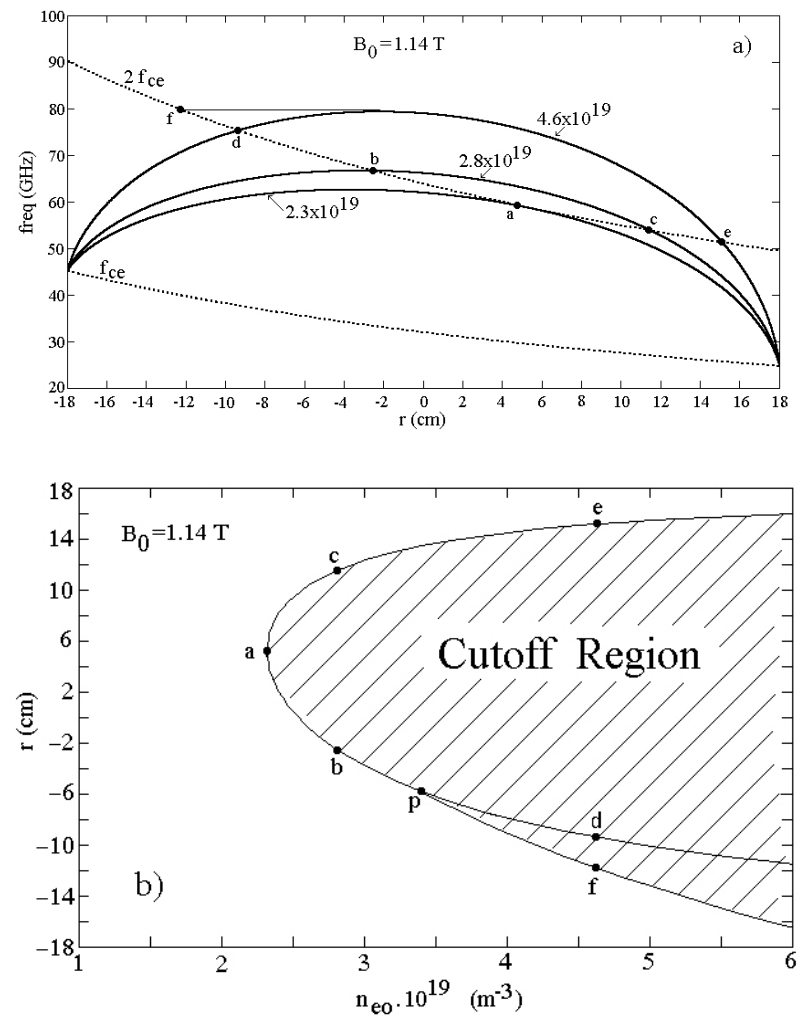

Figure 2. a. First and second ECE harmonics $\left(f_{c e}\right.$ and $\left.2 f_{c e}\right)$ and the right-hand cutoff frequencies were obtained for three different values of the electron density and for a toroidal magnetic field $B_{0}=1.14 T$. The point $a$ represents the position where the first cutoff occurs ( for the density $2.3 \times 10^{19} \mathrm{~m}^{-3}$ ). The frequencies between the points $b-c\left(\right.$ for $\left.n_{e 0}=2.8 \times 10^{19} \mathrm{~m}^{-3}\right)$ and $d-e$ ( for $n_{e 0}=4.6 \times 10^{19} \mathrm{~m}^{-3}$ ) are not accessible (non-local cutoff ). Also we can observe that the frequencies between the points $f-d\left(\right.$ for $n_{e 0}=4.6 \times 10^{19} \mathrm{~m}^{-3}$ ) are not externally accessible. b. The hachured region represents the region that is not accessible for the ECE X mode second harmonic radiation.

where $r_{f c 0}$ is the position at which the first cutoff occurs, for the situation when the density is rising. When the density falls, the position where the last ECE signal reappears also gives the $\alpha$ parameter. From this relation we see that, known the value $r_{f c 0}$, the $\alpha$ parameter can be estimated.

In Fig. 3.a, the Equation 9 is plotted for different values of $\alpha$. It is possible to that, the position of minimum density decreases with the increase of the value of $\alpha$. In Fig. 3.b, the value of $\alpha$ is plotted as a function of the first/last cutoff position, obtained from Equation 10.

In conclusion, the value for the $\alpha$ parameter and the peak density, $n_{e 0}$, of the density profile is uniquely obtained from the position(s) where the first/last ECE signal(s) cutoff occurs. 

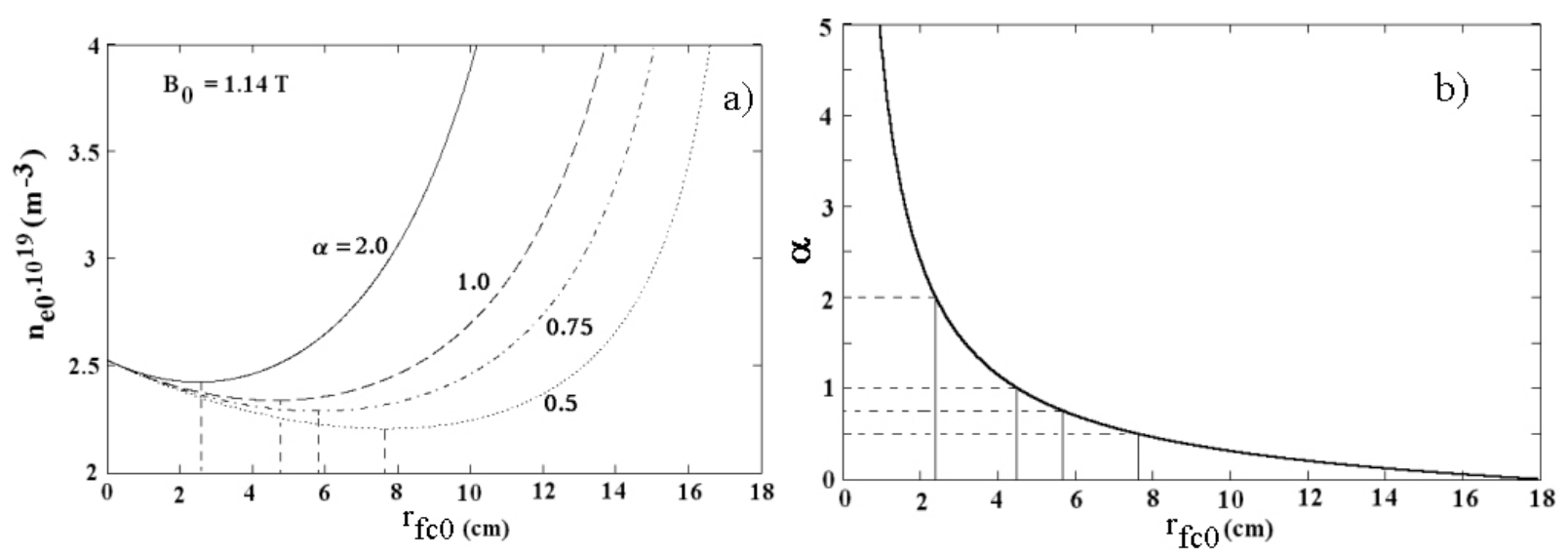

Figure 3. a. Central density as function of $r_{f c 0}$, obtained from Equation 9; b. The $\alpha$ parameter as function of the position $r_{f c 0}$, where the first/last cutoff occurs.

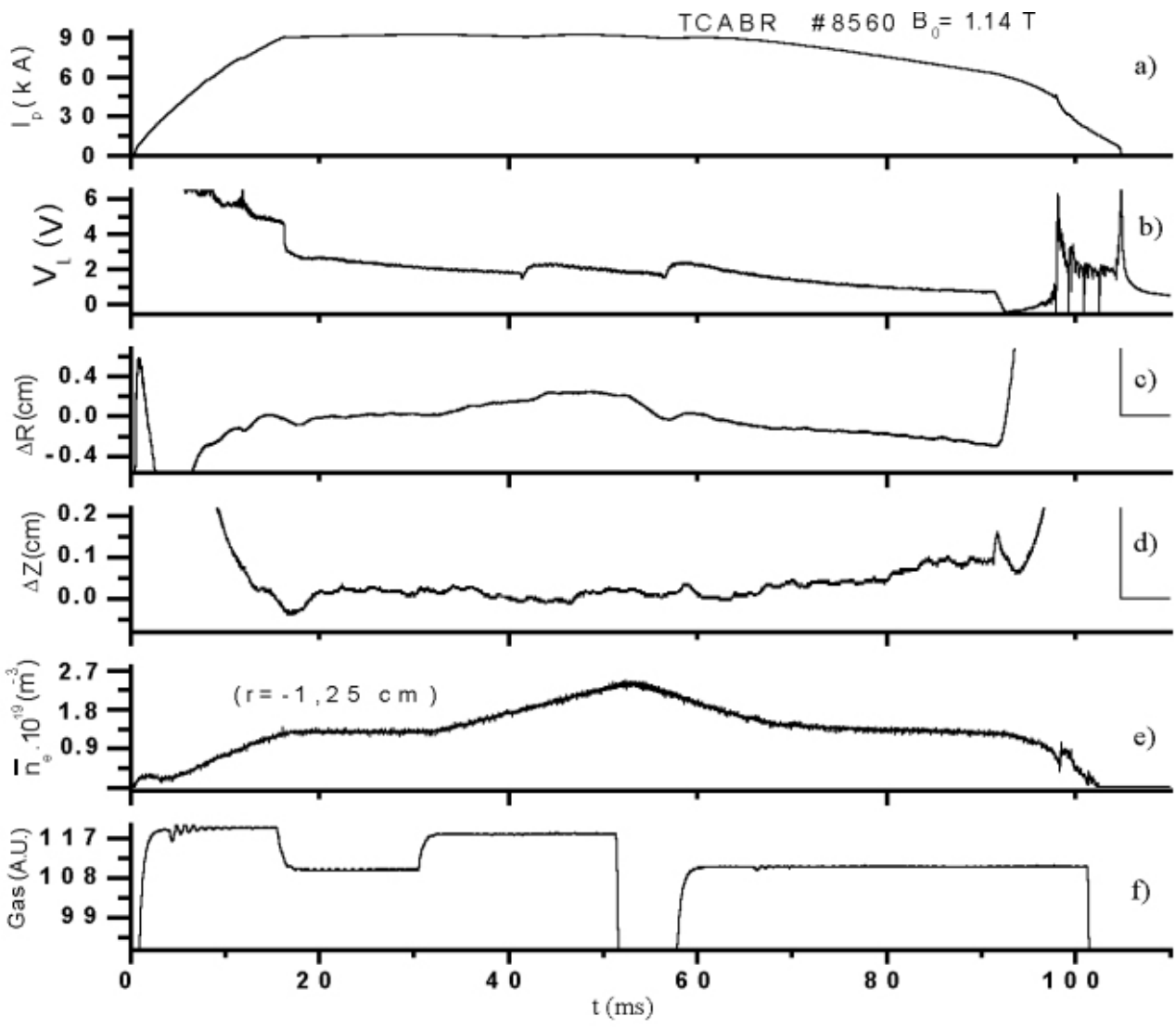

Figure 4. Time profiles obtained for the shot 8560 inTCABR tokamak: a. plasma current $I_{p} ;$ b. loop voltage $V_{L}$; c. horizontal plasma position $\Delta R$; d. vertical plasma position $\Delta Z$; e. line electron density at $r=-1.25 \mathrm{~cm} n_{e}$; $\mathbf{f}$. gas puffing control signal Gas. 


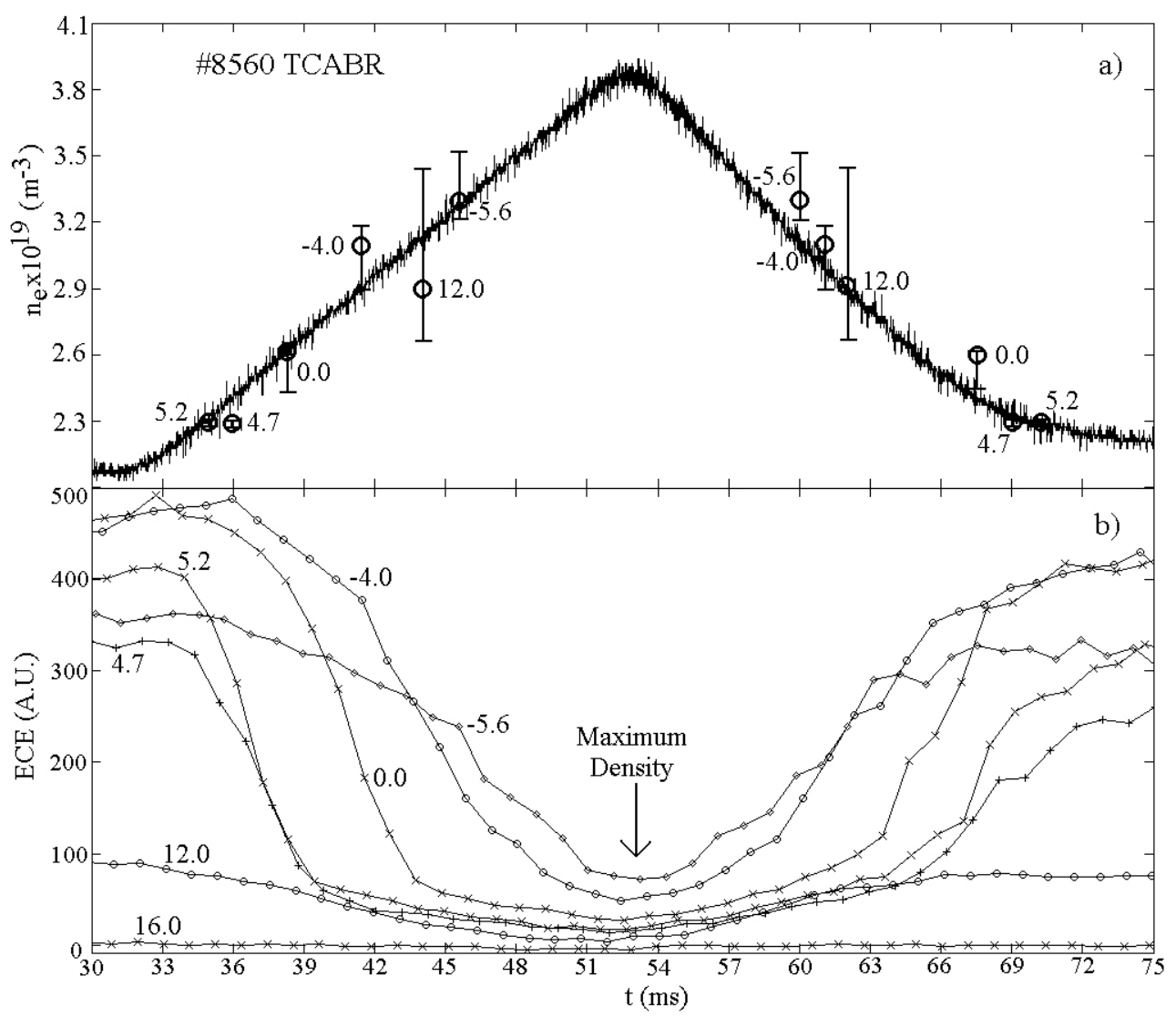

Figure 5. a. Electron peak density (continuous curve) and the electron density predicted by the ECE cutoffs (open circles); b. ECE radiation time profile during the right-hand cutoff for six different radial positions (in $\mathrm{cm}$ ).

\section{Experimental Results and Conclu- sions}

An external gas puffing injection system is usually used to control the electron density during the TCABR tokamak discharges. In Fig. 4, we present the following time profiles obtained from the thermal discharge 8560: a. plasma current $I_{p} ;$ b. loop voltage $V_{L} ;$ c. horizontal plasma position $\Delta R ;$ d. vertical plasma position $\Delta Z ;$; e. line electron density at $r=-1.25 \mathrm{~cm} n_{e} ;$; and f. gas puffing valve control signal Gas. For the discharge the plasma current was $I_{p} \approx 90 \mathrm{kA}$ ( at flat-top) and the total discharge duration was $t_{p} \approx 100 \mathrm{~ms}$. In Figs. 4.e and 4.f is possible to observe that, from $t \approx 30 \mathrm{~ms}$ to $t \approx 52 \mathrm{~ms}$, an increase of gas injection rate, externally imposed, causes a linear increase of the density. From $t \approx 52 \mathrm{~ms}$ to $t \approx 60 \mathrm{~ms}$ the gas injection valve is closed and a linear decrease in the density is observed. From $t \approx 60 \mathrm{~ms}$ up to the end of the discharge, the signal amplitude of the valve is adjusted to mantain the plasma density approximately constant. During the time interval of interest to our experiment (from 30 to $80 \mathrm{~ms}$ ) there is no considerable changes in the plasma position (horizontal or vertical).

In Fig. 5 the peak electron density and ECE signal from $t=30 \mathrm{~ms}$ to $t=75 \mathrm{~ms}$ is shown. In this time interval, de density rises linearly from $2.1 \times 10^{19} m^{-3}(t \approx$
$32 \mathrm{~ms}$ ) to $3.8 \times 10^{19} \mathrm{~m}^{-3}$ ( $t \approx 53 \mathrm{~ms}$ ). and after that, the electron density begins to decay reaching the value of $\approx 2.2 \times 10^{19} \mathrm{~m}^{-3}(t \approx 75 \mathrm{~ms})$. In Fig. 5.b, the ECE temporal profiles are presented for different positions in the plasma column. We observe that the emission from the positions $r=r_{f c 0} \approx 5.2 \mathrm{~cm}$ and $4.7 \mathrm{~cm}$ are first/last cutoff. Using those values in Equation 10 we obtain, for the $\alpha$ parameter, values between 0.86 and 0.97. After that, the electron density continues to rise and consequently more ECE channels are cutoff, one after another. The ECE radiation emitted from $r=16 \mathrm{~cm}$ was not cutoff, even at the highest density used in this experiment. The intersection point between the straight line determined at the flat top of the ECE signal with the straight line of maximum slope was used to determine the cutoff points. The vertical error bars in Fig. 5.a were obtained from the radiometer IF bandwidth $(\approx 2 \mathrm{GHz})$. Also, in these calculations we have made the assumption that the electron density profile does not change during the rising/falling of the electron density.

From Fig. 5.b we also observe that the cutoff in the ECE signal does not occurs instantaneously. The reason for this behavior can be explained by: a) the finite IF bandwidth; b) the dependence of the ECE signal on the reflection and refraction effects. These uncertainties lead to errors in the determination of electron density from ECE cutoffs. However, if we compare the density measurements, obtained with the interferometer, with those obtained from the ECE radia- 
tion right-hand cutoff ( points in Fig. 5.a) we observe a good agreement between them.

As conclusion, the main use of the ECE diagnostic is the electron temperature measurement. However when we are at the cutoff region, the ECE detection can be extended as an independent method to infer the electron density profile. This technique requires only the frequency calibration of the ECE radiometer and is useful if the density profile can be parameterized with two free parameters.

\section{Acknowledgements}

This work was partially supported by the Fundação de Amparo à Pesquisa do Estado de São Paulo ( FAPESP ).

\section{References}

[1] M. Bornatici, R. Cano, O. De Barbieri, and F. Engelman, Nuclear Fusion, 23, 1153 (1983).
[2] J. Lohr, Rev. Sci. Instrum., 59, 1608 (1988).

[3] D. C. Reddy and T. Eddington, Rev. Sci. Instrum., 67, 462 (1996).

[4] R. P. da Silva, A. M. M. Fonseca, J. H. Vuolo, E. R. Calderon, R. M. O. Galvão, Yu. K. Kuznetsov, J. C. Raffaelli, and the TCABR Team, "ECE Radiometry in the TCABR Tokamak" to be published in the Braz. J. of Phys. (2004).

[5] A. M. M. Fonseca, PhD. Thesis, Instituto de Física USP, São Paulo (2004).

[6] J. I. Elizondo, D. Korneev, I. C. Nascimento, and W. P. de Sá, Braz. J. of Phys. 32, 123 (2002).

[7] J. A. Bittencourt, Fundamentals of Plasma Physics (Third Edition, Edited and Published by the author), São José dos Campos, SP, Brasil, (2003). 